\title{
FORMAS DE APRENDIZAJE PARA MEJORAR LA INTERPRETACIÓN DE SITUACIONES PROBLÉMICAS
}

\section{WAYS OF LEARNING TO IMPROVE THE INTERPRETATION OF PROBLEM SITUATIONS}

\author{
Mawency Vergel Ortega ${ }^{1}$ \\ Luisa Stella Paz Montes² \\ Diego Mauricio Álvarez Paz ${ }^{3}$ \\ Universidad El Bosque- UFPS
}

\section{RESUMEN}

El desarrollo de habilidades en los diferentes escenarios pedagógicos se manifiesta cuando el estudiante se enfrenta a la resolución de ejercicios y problemas relacionados con los procesos de pensamiento matemático. Para la presente investigación se tomó como eje central el rendimiento escolar y el gusto por el aprendizaje de las matemáticas, con el fin de incursionar en nuevas técnicas o metodologías de mayor impacto en la asimilación de los conceptos de aprendizaje significativo, se desarrolló bajo la modalidad de investigación acción, El proceso enseñanza- aprendizaje a

$1 \quad$ Universidad Francisco de Paula Santander Cúcuta - Colombia, Orcid:0000-0001-8285-2968

Email: mawency@ufps.edu.co

2 Universidad Francisco de Paula Santander Cúcuta - Colombia, Orcid: 0000-0002-8887-3441

Email: luisastellapm@ufps.edu.co-Ipazmontes@gmail.com 3 Universidad El Bosque Bogotá - Colombia. Orcid. 0000-0001-6731-8417, Email: dmalvarezp@unbosque. edu.co-diegoalvarezp17@gmail.com través del uso de diferentes ayudas didácticas son una propuesta interdisciplinaria que permite trabajar los contenidos del lenguaje y las matemáticas de manera integradora. Estas estimulan el entusiasmo y la creatividad de los estudiantes al observar contenidos o temáticas, de una manera diferente, pero estricta y relacionando los contenidos con hechos cotidianos, $\mathrm{u}$ otorgando a las fracciones cualidades que permitan crear conexiones entre conceptos o procedimientos, típico de la matemática y características o acciones de las personas.

\section{PALABRAS CLAVE:}

aprendizaje, situaciones, interpretación, problemicas 


\section{ABSTRACT}

The development of skills in the different pedagogical scenarios is manifested when the student faces the resolution of exercises and problems related to mathematical thinking processes. For the present investigation, school performance and the taste for learning mathematics were taken as the central axis, in order to venture into new techniques or methodologies of greater impact on the assimilation of the concepts of meaningful learning, it was developed under the modality of action research, The teaching-learning process through the use of different teaching aids is an interdisciplinary proposal that allows to work the contents of language and mathematics in an integrative way. These stimulate the enthusiasm and creativity of the students by observing content or themes, in a different, but rigorous way, making use of the specific vocabulary of the topic and relating these contents to real situations or contexts, or giving the fractions human qualities that oblige to create connections between concepts or procedures, typical of mathematics and characteristics or actions of people.

\section{KEYWORDS:}

learning, situations, interpretation, problems

\section{INTRODUCCIÓN}

En el aula, hay marcadas diferencias en la forma y la velocidad con que algunos estudiantes captan los conceptos y avanzan sin ninguna dificultad, algunos discentes pueden tener dificultades que pueden ir desde la memorización de conceptos básicos hasta la representación de figuras geométricas, junto con la desconexión completa entre el conocimiento previo y los requeridos en el aula; entonces el niño encuentra nociones abstractas y deja de lado todo ese equipaje natural para recrear a través de las historias, juegos, dibujos entre otros, toda su capacidad narrativa e imaginativa, típica de su edad.

Los contenidos temáticos de la matemáticas, son necesariamente jerárquicos, el nuevo conocimiento depende de los previamente adquiridos, uno sobre la base del otro, por ejemplo en cuarto grado, la capacidad de comprender y aplicar las relaciones entre las unidades de los sistemas de medición, dependerá en gran medida del dominio que tenga para reconocer la línea recta, el plano cartesiano y, por lo tanto, los movimientos en el plano (rotación, traslación y reflexión, giros y desplazamiento). Esto significa que la tasa de aprendizaje está vinculada al nivel de apropiación de todos los conocimientos previos antes de ingresar nuevos.

En el transcurso de la investigación se enumeraron una serie de factores negativos influyentes, en el proceso de aprendizaje, entre los que destacan la dispersión en el aula, la poca atención y el compromiso con las actividades extracurriculares, las bajas calificaciones en el parcial y las evaluaciones bimestrales. Investigadores como Wagner, S. J. (1982) y Baroody, A. J.; Ginsburg, H. P. \& Waxman, B. (1983), han estado interesados en estudiar y demostrar que las matemáticas informales, son fundamentales para el enlace entre su conocimiento intuitivo y las matemáticas formales aprendidas en la escuela, en este mismo sentido Blythe, T. (1999), sugiere que para que los estudiantes logren un buen nivel de fluidez y retención de las habilidades o el contenido matemático que deben aprender, es necesario ofrecerles más y mejores oportunidades diseñadas para ponerlas en práctica; Por lo tanto, una de las principales dificultades en el "aprendizaje de las matemáticas" está relacionada con el lenguaje, ya que es a través de este se pueden interpretar los enfoques matemáticos y su correlación con la vida diaria que se vive fuera del aula. 
Allí se abre el espacio para usar diferentes técnicas tales como el juego, la narración, el dibujo, el cuento, el relato, de los temas, debido a su estructura secuencial-lineal y la forma lingüística que la memoria retiene sin demasiado esfuerzo, Marín, M. (2007), además de su semejanza por despertar emociones y sentimientos en el estudiante, conllevando a lograr una mejor interpretación de la situación . Se trata de proporcionar un contexto agradable y lúdico que estimule el aprendizaje de las matemáticas y desde ese entorno generar estrategias que permitan mejorar el rendimiento académico de los niños.

Se utilizó la metodología de investigaciónacción, y a través de esta se pudo obtener un diagnóstico de los inconvenientes y situaciones poco adecuadas, que entran en juego en el momento de orientar las clases de matemáticas y de los estudiantes recibir dichos conocimientos, es así como se hace necesario la implementación de estrategias pedagógicas que hagan el proceso más atractivo y ágil, logrando que el estudiante pueda asimilar de forma sencilla y rápida los diferentes conceptos,

Utilizar diferentes ayudas didácticas y estrategias metodológicas, es una excelente manera de estimular la participación de los estudiantes y profesores, brindando opciones de trabajo, utilizando el talento creativo que la mayoría de los niños tienen para escribir, comunicarse y aprender a través del lenguaje verbal y no verbal.

El trabajo de investigación, tiene como esencia central investigar y evaluar diferentes formas de aprendizaje que contribuyeron a mejorar los problemas de lectura-escritura, cálculo o interpretación de situaciones problemáticas a través de diferentes herramientas didáctica en el aula, para la enseñanza de las matemáticas en la educación primaria.

\section{MARCO TEÓRICO}

Es necesario recordar la importancia del papel mediador del docente, y todo su bagaje puesto a disposición de sus estudiantes, pues “.... la manera de aproximarnos a un problema, determina el éxito de su solución" Muñoz, A. (2014).

\subsection{La creatividad.}

La creatividad es definida por Muñoz , A. (2009), como "la capacidad que poseen las personas para producir ideas originales $\mathrm{y}$, a partir de estas, originar materiales nuevos, tomando siempre como referente el contexto social en el que tienen ocasión esas innovaciones -las cuales, en definitiva, comportan la expansión de los campos conceptuales y estéticos preexistentes".

La creatividad puede ser identificada como un proceso, como un producto, como una característica de la personalidad o como un fenómeno componencial en donde el contexto desempeña un papel importante. Pérez, J. (2005).

En este sentido, en el contexto escolar los profesores valoran más el esfuerzo que la habilidad. En otras palabras, mientras un estudiante espera ser reconocido por su capacidad (lo cual resulta importante para su estima), en el salón de clases se reconoce su esfuerzo.

\subsection{Estilos de aprendizaje}

Un estilo de aprendizaje es el conjunto de características, rasgos cognitivos, afectivos y fisiológicos que suelen expresarse conjuntamente cuando una persona debe enfrentar determinada situación. Estudios realizados han demostrado que los estudiantes tienen diferentes formas de aprender y que su rendimiento está asociado al estilo en que se le trasmitan los conocimientos. De acuerdo a lo 
anterior no existe un estilo mejor que otro, solo hay que identificar el estilo de cada individuo.

Lo primero que se debe hacer es conocer las falencias que presentan los estudiantes y sobre estas diseñar el proceso de enseñanza aprendizaje mas apropiado, reorientando las practicas pedagógicas utilizadas. Se debe tener presente que muchos estudiantes no le encuentran sentido a todo lo que en el aula se comunica sobre matemáticas o los problemas que allí se plantean.

La reingeniería en el proceso educativo es urgente toda vez que se hace necesario diseñar estrategias que potencialicen los aprendizajes, lo cual se conoce comúnmente como aprendizaje significativo. Tal y como dice Freire, P. (2003), "enseñar no se basa únicamente en transmitir conocimientos sino, más bien, de crear situaciones que favorezcan su construcción".

\section{METODOLOGÍA}

\subsection{Tipo de estudio.}

Se basa en los postulados de la investigaciónacción, definida por Latorre, A(2003) como "una indagación práctica realizada por el profesorado, de forma colaborativa, con la finalidad de mejorar su práctica educativa a través de ciclos de acción y reflexión" e igualmente se apoyó en la modalidad de proyecto factible. Es decir, la metodología fue el resultado de un conjunto de actividades parciales de reflexión y análisis que incidieron directamente en la planeación y desarrollo de nuevas situaciones previamente establecidas.

\subsection{Recolección de información.}

En el contexto de toda investigación, los instrumentos constituyen el elemento esencial para registrar la información que se obtiene durante la dinámica de la recolección de los datos. En palabras de Ruiz, C. (2002), los instrumentos de medición son procedimientos sistemáticos y estandarizados que permiten observar el comportamiento humano, con el fin de hacer inferencias sobre ciertas construcciones, características, dimensiones 0 atributos.

\subsection{Fases de la investigación.}

Teniendo en cuenta la naturaleza cíclica de la investigación-acción, se llevó a cabo una serie de fases de investigación que permitieron un elemento evaluativo entre ellas, y desde allí mejorar o repensar el curso a seguir, es decir, no son fases estáticas, pero deben cumplir con el rigor sistemático y científico utilizado en toda investigación cualitativa.

Fase 1. Diagnóstico. El rendimiento académico de los estudiantes se describe por períodos académicos, se realizaron encuestas acompañadas de la observación directa, para identificar las percepciones de los estudiantes respecto a la clase de matemáticas. Simultáneamente, se realizó una revisión documental para establecer experiencias exitosas que conllevaran a la construcción y enriquecimientos de las bases teóricas, así como a determinar los recursos y estrategias metodológicas más apropiadas.

Fase 2. Planificación y ejecución. Una vez identificado el recurso metodológico más asertivo se inició la fase de implementación del mismo, la cual comenzó con sesiones de capacitación que se realizaron con el apoyo de los maestros de narración oral, y guías de apoyo diseñadas con instrucciones específicas sobre cómo construir una historia usando términos y significados matemáticos, también se logró la construcción de ilustraciones y / o el uso de elementos de reciclaje para la elaboración de modelos.

Fase 3. Evaluación del recurso. Las narraciones o relatos historias se evaluaron adoptando la rúbrica para evaluar una narración ilustrada, 
bajo la licencia Creative Commons Attribution, e individualmente, se decodificaron las historias escritas en las variables de número de palabras, estructura, elementos de la narración, escritura e ilustración.

\section{RESULTADOS}

El analizar quiénes son los estudiantes y las formas en las que aprenden, permiten ayudar a los docentes $e$ instituciones educativas planificar acciones dirigidas para aumentar no sólo el rendimiento académico, sino mejorar los procesos de enseñanza aprendizaje que imparten los maestros en el aula de clase.

De allí que evolucionar constantemente en estos procesos, lleva consigo la necesidad de investigar el impacto e incidencia de cada una de estas herramientas o estrategias pedagógicas, sirven o no, cuál es su aceptabilidad y sobre todo, cómo hacer para que su implementación no se convierta en una tarea titánica y agotadora, sobrecargando al maestro de tareas y convirtiéndolas entonces en actividades negativas para el quehacer docente.

Se realizó un diagnóstico teniendo en cuenta los resultados académicos obtenidos por los estudiantes durante el primer bimestre lectivo, el cual se traduce en las calificaciones obtenidas en las diferentes evaluaciones tales como exámenes, quices y demás notas recolectadas por parte del docente, de los niños en la asignatura de matemática, por ser la más relevante al momento de tomar decisiones o resolver situaciones problémicas.

Se analizaron teniendo en cuenta la escala establecida, donde las calificaciones pueden ser baja, básica, alta o superior. En este orden de ideas, se analizaron los promedios correspondientes al primer bimestre académico, para la asignatura de matemáticas, donde aproximadamente el $50 \%$ de los estudiantes estaban en los niveles inferiores (bajo y básico).
Cuando se les pregunto a los niños por la causa de las bajas calificaciones en matemáticas, argumentaron que se debía a que la misma era "difícil" que debían estudiar o esforzarse mucho para entenderla además de que tenían que estar "concentrados" en clase.

Igualmente se evidenció que los niños están contentos con los profesores que le orientan esta asignatura, que se sienten motivados por el aprendizaje de la misma, los materiales y recursos que usan los docentes los incentivan a aprender, que les gustaría que algunas veces las clases se realizaran en otro lugar, distinto del salón y que fueran divertidas o "alegres". De igual forma comentaron sobre las evaluaciones parciales, afirman que son muy extensas y complejas de resolver, que pese a que estudian "bastante" les va mal en las calificaciones.

Cuando se les pregunto cómo les gustaría que se les "enseñara" los diferentes temas matemáticos, expresaron que no siempre se les explicara en el tablero, que los llevaran a biblioteca, fuera del salón de clase, que algunas veces se desarrollara a través de juegos, de cuentos, de narraciones, de crucigramas o de dibujos.

Teniendo en cuenta lo anterior y después de socializar los resultados con los docentes, se vió la necesidad de examinar las falencias, buscar e implementar estrategias que satisfagan las necesidades de un aprendizaje significativo, luego de una lluvia de ideas sobre las diferentes formas de impartir los conocimientos o dictar las clases y de evaluar las opciones expuestas, en consenso se decidió trabajar la propuesta de realizarlas a través de la utilización de cuentos o narraciones. Fue así como se acordó que como parte de la evaluación bimestral, los niños diseñaran un cuento, con uno de los temas vistos, para esta actividad se contó con la colaboración de los docentes y de un docente de narración oral quien brindo capacitación y todos 
los aspectos a tener en cuenta para realizar esta actividad.

Durante el proceso investigativo se realizaron cuatro ejercicios de creación de cuentos y uno final de picto-cuentos, donde satisfactoriamente se logró evidenciar mejoras en cada una de las entregas en cuanto redacción y estructuración de los cuentos, así como la utilización de los términos y conceptos matemáticos como parte de la historia contada y no como elemento aislado o sencillamente como nombre de los personajes; se pasa además de exposiciones muy pobres semánticamente a sustentaciones donde los niños exponen orgullosos sus actividades, demostrando total dominio de la temática y su aplicación en el contexto propio del cuento.

Al tiempo se logró recolectar un gran número de cuentos con elementos de la narración perfectamente definidos y sobre todo con un gran valor agregado de creatividad para el dibujo. Se estima que más del $80 \%$ de los cuentos cumple de forma alta y superior con la rúbrica de evaluación referenciada; de igual forma se encuentra creaciones muy bien diseñadas que de forma tangible evidencian la puesta en escena de toda la creatividad de los estudiantes. El picto-cuento final era de tema libre y cualquier tema visto en el año escolar era válido como eje central del mismo, pero a diferencia de los anteriores ejercicios acá se entregaron formatos pre-establecidos para el diseño de imágenes y redacción de ideas.

La actividad fue muy motivante para los estudiantes y los docentes, quienes decidieron implementarla, es así como las notas de los periodos académicos posteriores en el área de matemáticas, presentaron un incremento positivo significativo, además de que los estudiantes cambiaron la percepción que tenían de lo aburrida y tediosas que eran las matemáticas.

\section{CONCLUSIONES}

La investigación permitió evidenciar que es necesario que los docentes implementen diferentes prácticas pedagógicas al momento de orientar sus clases, pues muchas veces el bajo rendimiento de los estudiantes, así como su gusto por alguna materia, se debe a lo rutinario de las clases y que no se utilizan ayudas didácticas diferentes ni se experimenta o sugiere nuevas formas de aprendizaje.

La investigación generó una propuesta a nivel de conocimiento y metodológica que permitió conocer la problemática existente en la asignatura de matemáticas y la necesidad de implementar estrategias que mejoraran las habilidades lecto-escritoras, el aprendizaje significativo y sobre todo el aprovechamiento del talento innato para la construcción de cuentos e historias propias de la edad infantil., pues en muchos de los casos estas aptitudes se están subvalorando.

El entusiasmo que mostraron los estudiantes por participar de la experiencia, permitieron evidenciar que los cuentos como herramienta de aprendizaje son un elemento que motiva bastante. Las opiniones dadas por permitieron apreciar que a los niños les gusta contar sus propias historias y les parece divertido. EI trabajo mancomunado con los docentes del área de Lengua, hicieron posible que se pudiera trabajar de manera simultánea la competencia lingüística y la competencia matemática, tanto en la clase de matemáticas como en la clase de lengua castellana.

Pudimos apreciar que utilizar los cuentas para acentuar el aprendizaje es una propuesta que permite trabajar los contenidos de lengua y matemáticas de manera integradora.

Las valoraciones obtenidas por parte del docente de narración oral, también fueron muy enriquecedoras, se lograron obtener materiales de muy buena calidad, llevando consigo a 
plantear que el producto de este trabajo, recopila los cuentos más creativos y presenta un cuadernillo o librillo, que bajo la orientación de una nueva actividad extracurricular, los niños con todo aquello visto en clase durante todo el año, den rienda suelta a su creatividad e imaginación.

\section{REFERENCIAS BIBLIOGRÁFICAS}

Baroody, A. J.; Ginsburg, H. P. \& Waxman, B. (1983). Children's use of mathematical structure. Journal for Research in Mathematics Education, 14(3), 156-168.

Blythe, T. (1999). La enseñanza para la comprensión: guía para el docente. Buenos Aires: Paidós.

Flórez Ochoa, Rafael. (1997). Hacia una pedagogía del conocimiento. Bogotá: McGraw-Hill.

Freire, Paulo. (2003). Pedagogía de la autonomía: Saberes necesarios para la práctica educativa. España: Siglo XXI.

Latorre, Antonio (2003), La investigación-acción: Conocer y cambiar la práctica educativa. España, GRAÓ

Marín, M. (2007). Contar las matemáticas para enseñar mejor, Matematicalia: revista digital de divulgación matemática de la Real Sociedad Matemática Española, Vol. 3, No. 4-5.

Muñoz Sandoval, A. (2009).El desarrollo de las competencias en Educación Infantil. Propuestas y ejemplificaciones didácticas. Sevilla. Ed. MAD, S.L

Muñoz Sandoval, A. (2014).El desarrollo de las competencias en Educación Infantil. Propuestas y ejemplificaciones didácticas. Sevilla. Ed. MAD, S.L.

Pérez, J. J. (2005). Lectura y aprendizaje de las Matemáticas. Idea: revista de educación de Castilla-La Mancha, Toledo, año 1, n. 2, septiembre; p. 126-130

Rodríguez Cancio, M. (2011).Materiales y recursos en Educación Infantil. Manual de usos prácticos para el docente. Vigo. Ed: Ideas Propias.

Ruiz Bolívar, C. (2002). Instrumentos de Investigación Educativa. Venezuela: Fedupel.

Wagner, S. J. (1982). A Longitudinal Analysis of Ear-ly Number Concepts: From Numbers to Num-ber. Nueva York: Action and Thought 